DEPT. OF MATH/CMA. Univ. OF OSLO

Statistical Research Report No 5

ISSN 0806-3842 June 2008

\title{
PRICING BARRIER OPTIONS BY A REGIME SWITCHING MODEL
}

\author{
PÅL NICOLAI HENRIKSEN
}

\begin{abstract}
This paper introduces a new way of estimating parameters in a Brownian motion regime switching asset model to incorporate volatility clustering. The regime switching model is then applied to pricing of up-and-in barrier call options. We take probability of crossing the barrier between simulation points into account, and we increase accuracy in simulations by importance sampling. The regime switching model is compared to the Normal Inverse Gaussian model and the traditional Black and Scholes model, and option prices from the regime switching model is compared to the closed form expression of up-and-in barrier calls in a Black and Scholes market.
\end{abstract}

\section{IntRoduction}

Geometric Brownian motion or lognormal processes has been the standard model in financial mathematics since Black and Scholes [5]. In these models, one assumes that the logreturn of an asset has a normal distribution with a drift and a volatility term. In the literature, it has been displayed again and again that this model has very attractive mathematical features through closed form expressions. The lognormal assumptions is not bad, particularly when modeling on longer time intervals such as months or years. However, data from financial markets can easily be obtained on daily or even shorter intervals. The lognormal assumption is not as good on these intervals as the logreturns can experience distributional skewness and heavier tails than the normal distribution. Extensive work has been done to develop alternative methods that pick up these characteristics in short interval financial data. Regime switching models, introduced by Hamilton [14] in an autoregressive model setting, is a popular approach to capture these features. Frühwirth-Schnatter [13] is a good introduction to regime switching models - displaying several versions of regime switching and different areas of application. The Normal Inverse Gaussian (NIG) model, introduced in Barndorff-Nielsen [2], is another popular approach.

Financial data, especially on short time intervals, can experience discontinuities or jumps, e.g. positive jumps when companies land great contracts or negative jumps due to natural disasters such as earthquakes. Jump-diffusion models, for an introduction see e.g. Cont and Tankov [12], take this into account. The regime switching model in this

Date: June 24, 2008.

Key words and phrases. Regime Switching, Volatility clustering, Barrier Option, Autocovariance, Brownian Bridge, Importance sampling. 
paper, as is the case for the NIG model, does not take jumps into account explicitly, but incorporate possible jumps in the logreturn probability distribution.

For the numerical examples in this paper, we will assume three possible states of a stock value; one normal state, one state with steep increase in stock value (bull market) and one state with steep decrease in stock value (bear market). Letting the states be modeled by a Markov chain, one can assume geometric Brownian motion within each state to end up with a continuous regime switching model. Alexander [1], Brigo et al. [8] and Chourdakis $[10,11]$ are examples of continuous regime switching models.

One great challenge, especially for continuous models, is to take volatility clustering and autocovariance of size of asset returns into account. In this paper a new way of estimating parameters in the regime switching model is introduced. Through this estimation one may match autocovariance of squared asset returns in the model with autocovariance of squared returns from historical data.

One possible application of regime switching models is derivative pricing. Bollen [7], Hardy [15], Chourdakis [10] and Ching et al. [9] are examples of both exotic and non-exotic option pricing using continuous and non-continuous regime switching models. In this paper we will price up-and-in Barrier call options by Monte Carlo simulation of a continuous regime switching model. The pricing mechanism presented here will incorporate volatility clustering of the underlying asset, which may be particularly important for Barrier options as crossing a barrier is more likely if one is in a time period of high volatility. Further, probability of crossing the barrier between discontinuous simulation points may easily be included by a Brownian bridge. To increase the accuracy of the option prices, variance in the simulated option payoffs is reduced by importance sampling. We will compare the option prices from the regime switching model with option prices obtained by a closed form formula in a Black and Scholes market.

This paper is structured as follows: In Section 2 we argue for the reasoning behind the model and explain how the model is built; In Section 3 we display how the parameters in the model may be estimated so that the autocovariance of squared logreturns in the model coincide with the history of the underlying stock; In Section 4 we derive a risk neutral form of the asset price, present the up-and-in Barrier call option and display how this may be valued when incorporating a Brownian bridge and importance sampling; In Section 5 we look at numerical examples where we compare features of simulated and historical asset prices, compare the probability distribution of logreturns based on the NIG model and the regime switching model and compare option prices based on the regime switching model and the closed form Black and Scholes price. Finally, we conclude in Section 6.

\section{The Asset Model}

The regime switching model has been presented in various ways in the literature. Here we will present it with emphasis on the intuition behind the model. Throughout this paper, theory will be presented for an arbitrary number of states, but in our numerical examples we will use a three state model. In Boldin [6] one can find arguments for the three state 
model when analysing business cycles and with respect to estimation robustness. However, changing the number of states does not change complexity of the model.

In Figure 1 we have plotted the asset price of DNBNOR, the largest bank in Norway, in the period from February 2006 till February 2007. In the figure, it is clear that the asset price behaves normally in certain periods, marked by a white background, increases abnormally in periods, marked by a yellow background, and decreases abnormally in periods, marked by a red background. The three states in Figure 1 have been allocated subjectively by hand. In general, the state of the asset path at a given time is unobservable as one do not know if e.g. extreme asset dynamics is due to unlikely events in one state or more likely events in another state. In the regime switching model one usually assumes that the regime development over time may be modeled by a Markov chain. For a three state model this Markov chain is displayed in Figure 2 with different drift and volatility in each state. As we see, all states in the model are accessible at any time. The transition probabilities ${ }^{1}$ may be represented as:

$$
\boldsymbol{P}=\left[\begin{array}{cccc}
P_{11} & P_{12} & \cdots & P_{1 k} \\
P_{21} & P_{22} & \cdots & P_{2 k} \\
\vdots & \vdots & \ddots & \vdots \\
P_{k 1} & P_{k 2} & \cdots & P_{k k}
\end{array}\right]
$$

for $k$ possible states. The stationary distribution of the Markov chain is given by $\boldsymbol{\pi}=$ $\left[\pi_{1}, \pi_{2}, \cdots, \pi_{k}\right]$ such that

$$
\pi P=\pi .
$$

We will let the drift and volatility of an asset be determined by a Markov chain model without memory which is cadlag'. The Markov chain may have at most one transition between states within a short time interval $[t, t+d t)$. We will denote this Markov chain by $Y(t), Y(t) \in\{1,2, \cdots, k\}$.

Having introduced the $k$ state Markov chain, we let the asset dynamics be given by a traditional geometric Brownian motion within each state:

$$
\begin{aligned}
d S_{1}(t) & =\mu_{1} S(t) d t+\sigma_{1} S(t) d B(t) \\
d S_{2}(t) & =\mu_{2} S(t) d t+\sigma_{2} S(t) d B(t), \\
& \vdots \\
d S_{k}(t) & =\mu_{k} S(t) d t+\sigma_{k} S(t) d B(t),
\end{aligned}
$$

where $B$ represents Brownian motion and the subscripts represent the parameters in the different regimes. Thus, the asset dynamics may be represented as:

$$
d S(t)=S(t)\left(\sum_{i=1}^{k} \chi_{i}^{t}\left(\mu_{i}\right) d t+\sum_{i=1}^{k} \chi_{i}^{t}\left(\sigma_{i}\right) d B(t)\right),
$$

\footnotetext{
${ }^{1}$ These transition probabilities may easily be transferred to transition intensities in a continuous time Markov chain

${ }^{2} \mathrm{~A}$ function is cadlag if it is right-continuous with left limits. In our Markov chain model, cadlag simply means that the transitions between states are unpredictable.
} 


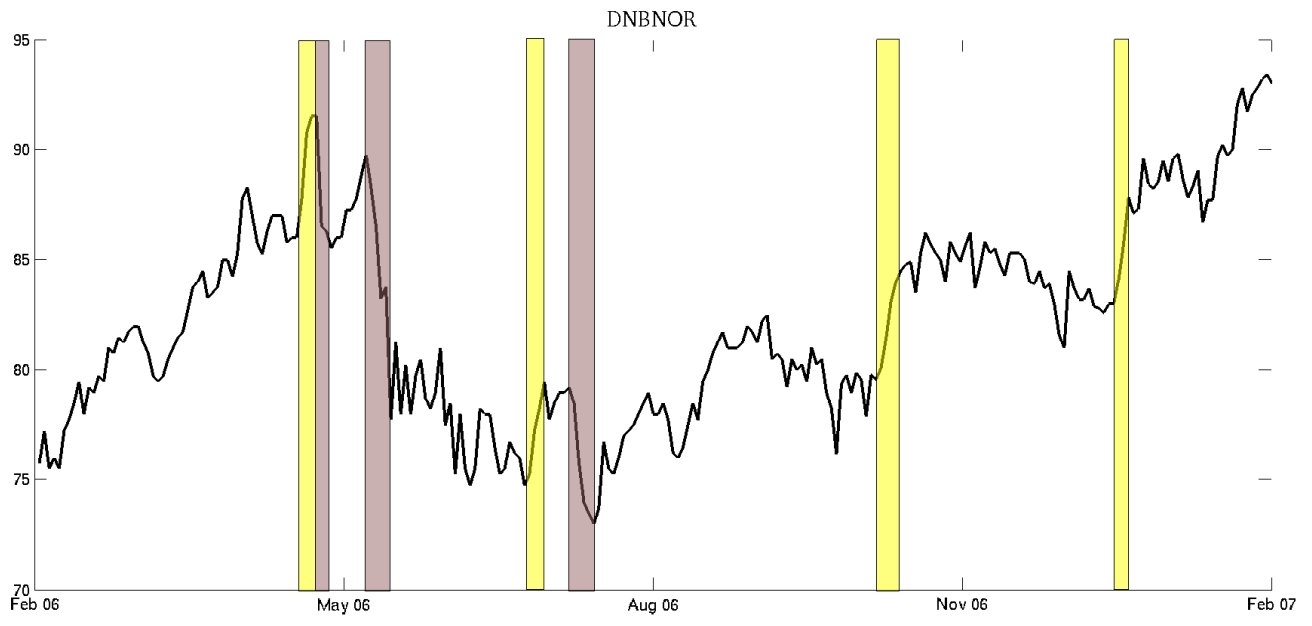

Figure 1. DNBNOR in the period from February 2006 till February 2007. Normal periods have been marked by a white background, periods with abnormal increase in asset price by a yellow background and periods with abnormal decrease by a red background.

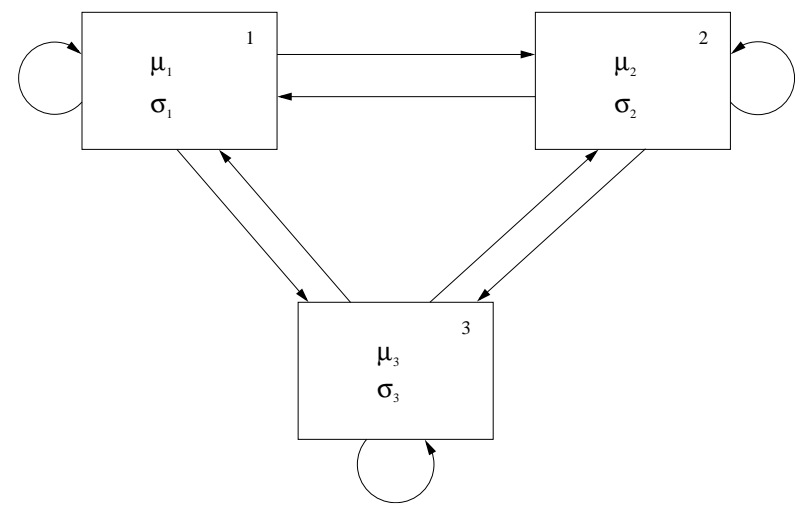

FigURE 2. Illustration of regime switching drift and volatility in a Markov chain with three states.

where $\chi$ is a indicator function to which state the Markov chain $Y(t)$ is in at a given time $t$. I.e.

$$
\chi_{1}^{t}=\left\{\begin{array}{ll}
1 & \text { if } Y(t)=1 \\
0 & \text { else }
\end{array}, \quad \chi_{2}^{t}=\left\{\begin{array}{ll}
1 & \text { if } Y(t)=2 \\
0 & \text { else }
\end{array}, \quad \cdots, \quad \chi_{k}^{t}=\left\{\begin{array}{ll}
1 & \text { if } Y(t)=k \\
0 & \text { else }
\end{array} .\right.\right.\right.
$$

We will assume that the starting point, $t=0$, of the Markov chain has distribution $\boldsymbol{\pi}$, which means that:

$$
\mathrm{E}\left(\chi_{1}^{t}\right)=\pi_{1}, \quad \mathrm{E}\left(\chi_{2}^{t}\right)=\pi_{2}, \quad \cdots \quad \mathrm{E}\left(\chi_{k}^{t}\right)=\pi_{k},
$$

for $t>0$. 
One may of course choose other processes than Brownian motion within each state, see e.g. Chourdakis [10] for jump diffusion processes or Rasmus [20] for NIG processes, but this makes the model more complex and decreases the intuitive tractability of the setup. It also makes parameter estimation even more challenging.

Proposition 2.1. Let the dynamics of some asset be given by equation (2.2). Then the asset price at time $t, S(t)$, is given by:

$$
\begin{array}{r}
S(t)=S(0) \cdot \exp \left(\int_{0}^{t} \sum_{i=1}^{k}\left(\chi_{i}^{s}\left(\mu_{i}-\frac{1}{2} \sigma_{i}^{2}\right)\right) d s\right) \\
\cdot \exp \left(\int_{0}^{t} \sum_{i=1}^{k}\left(\chi_{i}^{s}\left(\sigma_{i}\right)\right) d B(s)\right)
\end{array}
$$

where $B(s)$ is a Brownian motion, and $\mu_{i}, \sigma_{i}$ are constant drifts and volatilities within each state $i \in\{1,2, \cdots, k\}$ of a Markov chain with $k$ states.

Proof. Plugging (2.3) into Ito's formula immediately gives (2.2).

\section{Parameter estimation}

The most popular way of estimating the parameters in a regime switching model is by an EM algorithm based on the Baum-Welch algorithm for finding the parameters of a hidden Markov model, see e.q. Bilmes [4] for a thorough introduction. In this paper, we will use a somewhat different approach, where we estimate the parameters in two steps; one step to maximize the likelihood of a logreturn distribution and one step to match the autocovariance of squared logreturns. Since our parameter estimation technique focuses on the logreturn distribution and the autocovariance matching of squared logreturns, we will achieve better estimates regarding this than the EM algorithm.

For the parameter estimation purpose, we will assume that the logreturn model is based on an i.i.d. mix of normal distributions. This makes it easy to estimate the parameters $\mu_{i}$, $\sigma_{i}$ and $\pi_{i}$ for $i=1,2, \cdots, k$ based on e.g. daily financial data. The assumption that there is no state transition between data points applies. If the initial distribution of the mixture is given by $\boldsymbol{\pi}$, the probability distribution of logreturns at time $t$ is given by:

$$
f\left(x_{t}\right)=f\left(x_{t} \mid Y(t)=1\right) \cdot \pi_{1}+f\left(x_{t} \mid Y(t)=2\right) \cdot \pi_{2}+\cdots+f\left(x_{t} \mid Y(t)=k\right) \cdot \pi_{k} .
$$

Here $f\left(x_{t} \mid Y(t)=i\right), i=1,2, \cdots, k$, are normal distributions with parameters $\mu_{i}$ and $\sigma_{i}$. Thus, the parameters $\mu_{i}, \sigma_{i}$ and $\pi_{i}, i=1,2, \cdots, k$, may easily be estimated by maximum likelihood or equivalent methods. Using the maximum likelihood method, one has to numerically solve a set of nonlinear equations that maximizes

$$
\sum_{i=1}^{N} \ln \left(f\left(x_{i}\right)\right),
$$

where $x_{i}, i=1, \cdots, N$, are logreturns from financial data. 
The next step is to change the asset model from an i.i.d. mixture to a regime switching model based on an unobservable Markov chain as introduced in Section 2. This is done by estimating the transition probabilities $\boldsymbol{P}$. We want to use the flexibility of the transition probabilities to match the autocovariance of squared logreturns given the stationary distribution $\boldsymbol{\pi}$ found by maximum likelihood of (3.1). Based on ideas from Timmermann [21], we find the following expression for the autocovariance of squared logreturns from the regime switching model:

Proposition 3.1. Let $S_{\tau}$ be asset values at discrete times $\tau=\{\cdots, t-\Delta t, t, t+\Delta t, \cdots\}$ and let the logreturn be defined as $R_{t}=\ln \frac{S_{t}}{S_{t-\Delta t}}$. Then the autocovariance of $R_{t}^{2}$ is given by

$$
\operatorname{cov}\left(R_{t}^{2}, R_{t+s \Delta t}^{2}\right)=E\left[R_{t}^{2} R_{t+s \Delta t}^{2}\right]-\mu_{R_{t}^{2}} \mu_{R_{t+s \Delta t}^{2}},
$$

for lags $s \in \mathbb{N}$. If $S(t)$ is modeled by regime switching with $k$ regimes and no possible regime switch in the periods $[t, t+\Delta t)$, the terms in (3.2) are given by:

$$
\begin{aligned}
E\left[R_{t}^{2} R_{t+s \Delta t}^{2}\right] & =\sum_{i=1}^{k} \sum_{j=1}^{k} \pi_{i} P_{i j}^{(0, s)} E\left[\kappa_{i}^{t} \kappa_{j}^{t+s \Delta t}\right], \\
\mu_{R_{t}^{2}} & =\sum_{i=1}^{k} \pi_{i} E\left[\kappa_{i}^{t}\right], \\
\mu_{R_{t+s \Delta t}^{2}} & =\sum_{i=1}^{k} \sum_{j=1}^{k} \pi_{i} P_{i j}^{(0, s)} E\left[\kappa_{j}^{t}\right],
\end{aligned}
$$

where

$$
\kappa_{i}^{t}=\left(\mu_{i}-\frac{1}{2} \sigma_{i}^{2}\right)^{2} \Delta t^{2}+2 \sigma_{i}\left(\mu_{i}-\frac{1}{2} \sigma_{i}^{2}\right)(B(t)-B(t-\Delta t)) \Delta t+\sigma_{i}^{2}(B(t)-B(t-\Delta t))^{2},
$$

and $\boldsymbol{P}^{(0, s)}=\boldsymbol{P}^{s}$, and the expectations are given by

$$
E\left[\kappa_{i}^{t} \kappa_{j}^{t+s \Delta t}\right]= \begin{cases}\left(\mu_{i}-\frac{1}{2} \sigma_{i}^{2}\right)^{4} \Delta t^{4}+6\left(\mu_{i}-\frac{1}{2} \sigma_{i}^{2}\right)^{2} \sigma_{i}^{2} \Delta t^{3}+3 \sigma_{i}^{4} \Delta t^{2} & s=0 \\ \left(\mu_{i}-\frac{1}{2} \sigma_{i}^{2}\right)^{2}\left(\mu_{j}-\frac{1}{2} \sigma_{j}^{2}\right)^{2} \Delta t^{4}+\left(\mu_{i}-\frac{1}{2} \sigma_{i}^{2}\right)^{2} \sigma_{j}^{2} \Delta t^{3}+\cdots & \\ \left(\mu_{j}-\frac{1}{2} \sigma_{j}^{2}\right)^{2} \sigma_{i}^{2} \Delta t^{3}+\sigma_{i}^{2} \sigma_{j}^{2} \Delta t^{2} & s \geq 1\end{cases}
$$

and

$$
E\left[\kappa_{i}^{t}\right]=\left(\mu_{i}-\frac{1}{2} \sigma_{i}^{2}\right)^{2} \Delta t^{2}+\sigma_{i}^{2} \Delta t
$$

Proof. The logreturn may be expressed as

$$
R_{t} \stackrel{d}{=} \sum_{i=1}^{k} \chi_{i}^{t-\Delta t}\left(\left(\mu_{i}-\frac{1}{2} \sigma_{i}^{2}\right) \Delta t+\sigma_{i} B(\Delta t)\right)
$$


which gives the square logreturn as

$$
R_{t}^{2} \stackrel{d}{=} \sum_{i=1}^{k} \chi_{i}^{t-\Delta t}\left(\kappa_{i}^{t}\right) .
$$

Taking conditional expectations on regime state at time $t-\Delta t$ we end up with (3.3), (3.4) and (3.5). The expectations may easily be found by direct calculation using the following properties of the Brownian motion:

$$
\begin{aligned}
E[B(\Delta t)] & =0, & & E\left[B(\Delta t)^{2}\right]=\Delta t, \\
E\left[B(\Delta t)^{3}\right] & =0, & & E\left[B(\Delta t)^{4}\right]=3 \Delta t^{2},
\end{aligned}
$$

and independence and stationarity of increments.

Having established the relationship between $\boldsymbol{P}$ and the autocovariance of square logreturns in Proposition 3.1, the only thing remaining to match square logreturn autocovariance from data is restrictions to make sure that $\boldsymbol{P}$ satisfies (2.1) and probability properties. For $k=3$ regimes these restrictions can be expressed as:

$$
\begin{aligned}
& P_{13}=P_{21} \frac{\pi_{2}}{\pi_{1}}+P_{31} \frac{\pi_{3}}{\pi_{1}}-P_{12} \\
& P_{23}=P_{12} \frac{\pi_{1}}{\pi_{2}}+P_{32} \frac{\pi_{3}}{\pi_{2}}-P_{21} \\
& P_{11}=1-P_{21} \frac{\pi_{2}}{\pi_{1}}-P_{31} \frac{\pi_{3}}{\pi_{1}} \\
& P_{22}=1-P_{12} \frac{\pi_{1}}{\pi_{2}}-P_{32} \frac{\pi_{3}}{\pi_{2}} \\
& P_{33}=1-P_{32}-P_{31}
\end{aligned}
$$

This leaves us with four unknowns, $P_{12}, P_{21}, P_{31}$ and $P_{32}$, which have to be found numerically under the constrain that $0 \leq P_{i j} \leq 1$ for $i, j \in\{1,2,3\}$.

The final issue remaining for this parameter estimation to be valid, is to verify that the introduction of regime switching through $\boldsymbol{P}$ does not significantly alter the logreturn distribution compared to (3.1). It is natural that the logreturn distribution under regime switching takes another expression than (3.1) as one would be dependent on which regime the Markov chain is in at any given time. However, if the initial distribution of the Markov chain is given by $\boldsymbol{\pi}$ and simulations are done over an adequately long time period, one would expect logreturns from the regime switching model to be distributed similarly to (3.1). We will see in the numerical examples in Section 5 that this is the case.

\section{Option PRICING}

In Rasmus [20] a theory for barrier option pricing in a regime switching setting has been initialized, but not completed due to time constrains. In this section we extend this work to price Barrier options by Monte Carlo simulations when incorporating; (i) volatility clustering through the matching of the autocovariance of squared historical logreturns as described in Section 3, (ii) the probability of crossing the barrier between simulation points, 
(iii) variance reduction by importance sampling. Throughout the section, we will stick to the up-and-in Barrier call option, but the methods presented are general and can be applied to other Barrier option types and also extended to other path dependent options.

The price $\Pi$ of an up-and-in barrier call option with barrier $B$ and strike $K$ may be written as:

$$
\Pi=e^{-r T} \mathrm{E}_{\mathrm{Q}}[g(\boldsymbol{X})]=e^{-r T} \mathrm{E}_{\mathrm{Q}}\left[(S(T)-K)^{+} \chi\left(\sup _{0 \leq t \leq T} S(t)>B\right)\right],
$$

where $T$ is time to maturity, $r$ is a risk free constant discount rate and $g(\boldsymbol{X})$ is the payoff as a function of some Lévy process - in our case the regime switching process. We see that the option has payoff $\max (S(T)-K, 0)$ if the asset price under a risk neutral probability $\mathrm{Q}$ crosses the barrier $B$ at any time $0 \leq t \leq T$.

When the asset price $S(t)$ is modeled by a Black and Scholes market the option price (4.1) can be written in closed form, see e.g. Hull [17] chapter 19, as

$$
\begin{aligned}
\Pi_{B \& S} & =S_{0} N\left(x_{1}\right)-K e^{-r T} N\left(x_{1}-\sigma \sqrt{T}\right)-S_{0}\left(\frac{B}{S_{0}}\right)^{2 \lambda}\left(N(-y)-N\left(-y_{1}\right)\right) \\
& +K e^{-r T}\left(\frac{B}{S_{0}}\right)^{2 \lambda-2}\left(N(-y+\sigma \sqrt{T})-N\left(-y_{1}+\sigma \sqrt{T}\right)\right),
\end{aligned}
$$

where $S_{0}$ is the initial asset price, $\sigma$ is the asset volatility, $N$ is the standard normal cumulative distribution function and

$$
\begin{aligned}
\lambda & =\frac{r+\sigma^{2} / 2}{\sigma^{2}}, \\
y & =\frac{\ln \left(B^{2} /\left(S_{0} K\right)\right)}{\sigma \sqrt{T}}+\lambda \sigma \sqrt{T}, \\
y_{1} & =\frac{\ln \left(B / S_{0}\right)}{\sigma \sqrt{T}}+\lambda \sigma \sqrt{T}, \\
x_{1} & =\frac{\ln \left(S_{0} / B\right)}{\sigma \sqrt{T}}+\lambda \sigma \sqrt{T} .
\end{aligned}
$$

We will use this option price formula for comparison in Section 5.

Based on the regime switching model, we will simulate option prices based on (4.1). Hence, we need a risk neutral measure Q. Proposition 4.1 presents one possible choice of risk neutral measure which is an analog to the Black \& Scholes measure for the regime switching setting with $k$ regimes. This risk neutral measure has also been applied in Bollen [7], Hardy [15], Chourdakis [10] and Ching et al. [9].

Proposition 4.1. Let $S(t)$ have dynamics given by equation (2.2), where $B(t)$ is a Brownian motion under $\mathrm{P}$, and $\mu_{i}$ and $\sigma_{i}$ are constant drift and volatility in state $i$. For $t \leq T$ 
let

$$
\begin{aligned}
M_{t}= & \exp \left(\int_{0}^{t} \sum_{i=1}^{k} \chi_{i}^{s}\left(-\frac{1}{2} \frac{\left(\mu_{i}-r\right)^{2}}{\sigma_{i}^{2}}\right) d s\right) \\
& \cdot \exp \left(\int_{0}^{t} \sum_{i=1}^{k} \chi_{i}^{s}\left(-\frac{\mu_{i}-r}{\sigma_{i}}\right) d B(s)\right)
\end{aligned}
$$

and

$$
d \mathrm{Q}=M_{T} d \mathrm{P} .
$$

Since $M_{t}$ is a martingale (w.r.t. $\left.\mathrm{P}\right), \mathrm{Q}$ is a probability measure and the process

$$
d W(t)=d B(t)+\sum_{i=1}^{k} \chi_{i}^{t}\left(\frac{\mu_{i}-r}{\sigma_{i}}\right) d t
$$

is a Brownian motion under Q. Further, since

$$
S(t)=S(0) \cdot \exp \left(\int_{0}^{t} \sum_{i=1}^{k} \chi_{i}^{s}\left(r-\frac{1}{2} \sigma_{i}^{2}\right) d s+\int_{0}^{t} \sum_{i=1}^{k} \chi_{i}^{s}\left(\sigma_{i}\right) d W(s)\right)
$$

$e^{-r t} S(t)$ is a martingale (w.r.t. $\mathrm{Q}$ ) and $\mathrm{Q}$ is a equivalent martingale measure.

Proof. Since

$$
\mathrm{E}\left[\exp \left(\frac{1}{2} \int_{0}^{T} \sum_{i=1}^{k} \chi_{i}^{s}\left(\frac{\left(\mu_{i}-r\right)^{2}}{\sigma_{i}^{2}}\right) d s\right)\right]<\infty
$$

$M_{t}$ is a martingale by the Novikov condition.

Further, by comparing expression (2.3) with (4.4) and taking into account the dynamics $(2.2)$, it is easily seen that the dynamics of $S_{d}(t)=\exp (-r t) S(t)$ is given by:

$$
d S_{d}(t)=\sum_{i=1}^{k} \chi_{i}^{t}\left(\sigma_{i}\right) S(t) d W(t)
$$

Thus, there is no drift term in the dynamics, and $S_{d}(t)$ must be a martingale.

The rest of the proof is in line with the proof of the Girsanov theorem (see Øksendal [19]).

To simulate risk neutral regime switching asset prices, we may in practice simulate the Markov chain regimes prior to the asset price simulation. This is because the Markov chain is independent of the Brownian motion in the asset price. This gives us a set of regime switching times $t_{1}, \cdots, t_{n-1}$ with initial time $t_{0}=0$ and maturity $t_{n}=T$ with corresponding regimes $Y_{0}, Y_{1}, \cdots, Y_{n-1}{ }^{3}$. This means that to simulate one asset path, we do not have to simulate $T / \Delta t$ Brownian motion points, as the regime remains constant in

\footnotetext{
${ }^{3}$ Note that simulation from a continuous Markov chain leaves this setup unchanged.
} 
the period $\left[t_{m-1}, t_{m}\right), m=1, \cdots, n$. Thus, $n$ simulation points is adequate. For the risk neutral asset price, this gives the following simulations:

$$
S\left(t_{m}\right)=S\left(t_{m-1}\right) \cdot \exp \left(\left(r-\frac{1}{2} \sigma_{Y_{m-1}}^{2}\right)\left(t_{m}-t_{m-1}\right)+\sigma_{Y_{m-1}} \sqrt{t_{m}-t_{m-1}} z_{m}\right),
$$

for $m=1, \cdots, n$ where $\boldsymbol{z}=\left(z_{1}, \cdots, z_{n}\right)$ is i.i.d. simulations from a standard normal distribution.

One remaining challenge, is that the asset path may cross the barrier $B$ at some point between two simulations points $S\left(t_{j-1}\right)$ and $S\left(t_{j}\right)$ (which both are below the barrier). Since we know that the regime switching model experiences Brownian motion properties between simulation points, we may use expressions for Brownian bridges from Glasserman [16] chapter 6 or Joshi and Leung [18]. These expressions give us the probability $P_{\text {crossing }}$ of crossing the barrier between the simulations points $S\left(t_{j-1}\right)$ and $S\left(t_{j}\right)$ :

$$
P_{\text {crossing }}=\exp \left(-\frac{2\left(\ln (B)-\ln S\left(t_{j-1}\right)\right)\left(\ln (B)-\ln \left(S\left(t_{j}\right)\right)\right.}{\left(t_{j}-t_{j-1}\right) \sigma_{Y_{j-1}}^{2}}\right) .
$$

One very useful tool to reduce variation in simulated option payoffs, especially when the barrier $B$ and/or the strike $K$ is much greater than the initial asset price $S(0)$, is importance sampling (IS). A general introduction to importance sampling can be found in Glasserman [16]. The main idea of IS is to sample from the region where things happen. In an option pricing setting this means sampling in the region where the option is in the money, as there is little information in many simulations with zero payoff. Here, we will perform IS by changing the drift of the underlying risk neutral asset such that the probability of crossing the barrier increases. If we look at (4.5), we see that this may be written as

$$
S\left(t_{m}\right)=S\left(t_{m-1}\right) \cdot \exp \left(X_{m}\right)
$$

where $X_{m} \sim N\left(\mu_{m}, \sigma_{m}^{2}\right)$ and

$$
\begin{aligned}
\mu_{m} & =\left(r-\frac{1}{2} \sigma_{Y_{m-1}}^{2}\right)\left(t_{m}-t_{m-1}\right), \\
\sigma_{m}^{2} & =\sigma_{Y_{m-1}}^{2}\left(t_{m}-t_{m-1}\right) .
\end{aligned}
$$

Since importance sampling is done by changing the drift, we choose to sample from $X_{m} \sim$ $N\left(\mu_{m}+\mu_{\mathrm{IS}}, \sigma_{m}^{2}\right)$. Denote this importance sampling probability measure by Q + IS, and let $\mu_{\text {IS }}$ be determined such that

$$
\mathrm{E}_{\mathrm{Q}+\mathrm{IS}}[S(T)]=B
$$

This gives

$$
\mu_{\mathrm{IS}}=\frac{\ln B-\ln S(0)}{T}-r
$$

Under the importance sampling probability measure, the option price (4.1) takes the form

$$
\Pi=e^{-r T} \cdot \mathrm{E}_{\mathrm{Q}+\mathrm{IS}}\left[g(\boldsymbol{X}) \cdot \frac{d \mathrm{Q}}{d(\mathrm{Q}+\mathrm{IS})}\right] .
$$


Here $\boldsymbol{X} \sim N\left(\boldsymbol{\mu}+\boldsymbol{\mu}_{\mathrm{IS}}, \boldsymbol{\sigma}\right)$, where $\boldsymbol{\mu}=\left[\mu_{1}, \cdots, \mu_{n}\right]^{\prime}, \boldsymbol{\mu}_{\mathrm{IS}}=\mathbf{1} \cdot \mu_{\mathrm{IS}}, \boldsymbol{\sigma}=\operatorname{diag}\left(\sigma_{1}, \cdots, \sigma_{n}\right)$ and $\frac{d \mathrm{Q}}{d(\mathrm{Q}+\mathrm{IS})}$ is the Radon-Nikodym derivative ${ }^{4}$ given by

$$
\begin{aligned}
\frac{d \mathrm{Q}}{d(\mathrm{Q}+\mathrm{IS})} & =\prod_{m=1}^{n} \frac{\frac{1}{\sqrt{2 \pi} \sigma_{m}} \exp \left(-\frac{1}{2}\left(\frac{X_{m}-\mu_{m}}{\sigma_{m}}\right)^{2}\right)}{\frac{1}{\sqrt{2 \pi} \sigma_{m}} \exp \left(-\frac{1}{2}\left(\frac{X_{m}-\left(\mu_{m}+\mu_{\mathrm{IS}}\right)}{\sigma_{m}}\right)^{2}\right)} \\
& =\exp \left(-\sum_{m=1}^{n} \frac{X_{m} \mu_{\mathrm{IS}}}{\sigma_{m}^{2}}+\frac{1}{2} \sum_{m=1}^{n} \frac{2 \mu_{m} \mu_{\mathrm{IS}}+\mu_{\mathrm{IS}}^{2}}{\sigma_{m}^{2}}\right)
\end{aligned}
$$

Using the framework introduced above, we can now price up-and-in barrier call options incorporating volatility clustering in the underlying asset, taking into account the possibility of crossing the barrier between simulation points and increasing efficiency in the simulations by importance sampling.

\section{Numerical examples}

In this section we will apply the methods presented earlier to the main index on the Norwegian Stock exchange OBX. First we will estimate the parameters in the three regime distribution based on daily data and compare the goodness of fit of the distribution with the lognormal and the NIG distributions. Thereafter, up-and-in call options will be valuated based on simulations and closed form formulas for the regime switching model and the Black and Scholes model respectively.

We have daily data for OBX in the period from February 2002 to February 2007. Based on these we estimate parameters for the regime switching distribution by maximizing the likelihood and matching autocovariance as described in Section 3. The resulting distribution, given by expression (3.1), is compared to a normal distribution, for which the parameters are estimated by the mean and standard deviation of the logreturns, and a NIG distribution. The parameters of the NIG distribution $\left(\alpha_{\mathrm{NIG}}, \beta_{\mathrm{NIG}}, \mu_{\mathrm{NIG}}, \delta_{\mathrm{NIG}}\right)$ are estimated by:

$$
\begin{aligned}
\alpha_{\mathrm{NIG}} & =\sqrt{\frac{3 K-4 S^{2}-9}{V\left(K-\frac{5}{3} S^{2}-3\right)^{2}}}, \\
\beta_{\mathrm{NIG}} & =\frac{S}{\sqrt{V}\left(K-\frac{5}{3} S^{2}-3\right)}, \\
\mu_{\mathrm{NIG}} & =M-\frac{3 S \sqrt{V}}{3 K-4 S^{2}-9}, \\
\delta_{\mathrm{NIG}} & =3^{3 / 2} \frac{\sqrt{V\left(K-5 / 3 S^{2}-3\right)}}{\left(3 K-4 S^{2}-9\right.},
\end{aligned}
$$

\footnotetext{
4 also called the likelihood ratio
} 


\begin{tabular}{|c|c|c|c|c|c|c|c|c|c|c|c|c|}
\hline \multirow{2}{*}{$\frac{\text { Re. sw. }}{\text { OBX }}$} & $\mu_{1}$ & $\sigma_{1}$ & $\pi_{1}$ & \multicolumn{2}{|c|}{$\mu_{2}$} & \multicolumn{2}{|c|}{$\sigma_{2}$} & \multicolumn{2}{|c|}{$\pi_{2}$} & $\mu_{3}$ & $\sigma_{3}$ & $\pi_{3}$ \\
\hline & 0.0063 & 0.0070 & 0.3669 & & 0029 & $0.0^{\circ}$ & 217 & 0.20 & & -0.0024 & 0.0088 & 0.4322 \\
\hline & & NIG & $\alpha_{\mathrm{NI}}$ & & $\beta_{\mathrm{NI}}$ & & $\mu_{\mathrm{N}}$ & JIG & $\delta_{\mathrm{N}}$ & & & \\
\hline & & OBX & \begin{tabular}{|l|l|} 
& 84.0
\end{tabular} & & -12.6 & 123 & 0.0 & 027 & 0.01 & 133 & & \\
\hline & & & Nor & mal & $\mu_{\mathrm{Nol}}$ & mal & $\sigma_{\mathrm{No}}$ & rmal & & & & \\
\hline & & & $\mathrm{OB}$ & & 0.00 & 07 & 0.0 & 128 & & & & \\
\hline
\end{tabular}

TABLE 1. The estimated parameters for the three state regime switching distribution (or rather the i.i.d. normal mixture distribution), the NIG distribution and the normal distribution for OBX daily logreturns.

where $M, V, S$ and $K$ are the mean, variance, skewness and kurtosis of the logreturns. See e.g. Benth [3] for an introduction to the NIG distribution and estimation in a financial setting. Resulting parameters can be found in Table 1.

From the parameters of the three state regime switching model (or rather the i.i.d. normal mixture), we see that there are no particularly extreme states. The stationary distribution $\boldsymbol{\pi}$ indicates that all the states contribute in a fairly high proportion of the time. Further, none of the three drifts, $\mu_{i}$ with $i \in\{1,2,3\}$, are very extreme. The positive drift is highest in absolute value, but the volatility of the state with most negative drift is much greater than the other volatilities. This gives a skew distribution to the left, which is consistent with the $\beta_{\mathrm{NIG}}$ parameter. Comparing the regime switching parameters with the normal parameters, we see that the regime switching model succeeds in picking up the kurtosis in the logreturn distribution. All of these observations can be recognized in Figure 3.

As noted in the end of Section 3, we need to make sure that the introduction of regime switching through the transition probabilities $\boldsymbol{P}$ does not make the logreturn distribution from the regime switching model significantly different to the probability distribution of the i.i.d. normal mixture given by Equation (3.1). In Figure 3 the i.i.d. normal mixture distribution is given by the whole line and the probability distribution based on 10000 simulations of 250 daily logreturns from the regime switching model is given by the stars. There is no significant difference between the distributions, which validates the OBX regime switching parameter estimation.

It is not immediately obvious whether the NIG distribution or the regime switching distribution gives the best fit to data in Figure 3. It can therefore be helpful to look at the loglikelihoods in Table 2 as a measure of goodness of fit. Here we see that the regime switching distribution (or rather the i.i.d. mixture distribution) has the highest loglikelihood, closely followed by the NIG distribution. Again referring to Figure 3, it 

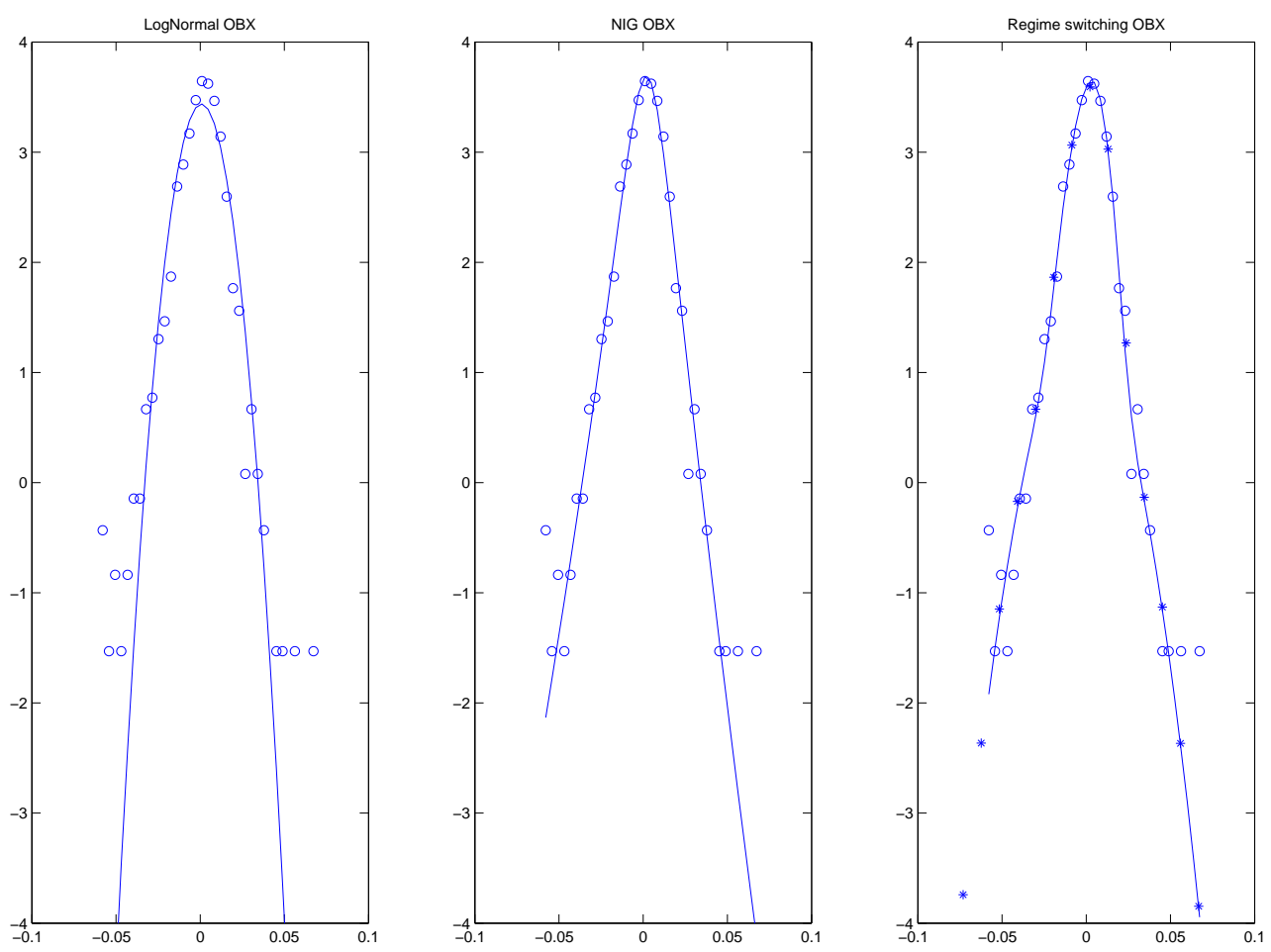

FIGURE 3. Log probability for the normal distribution, the NIG distribution and the regime switching distribution (or rather the i.i.d. normal mixture distribution) with parameters in Table 1 . The circles represents the corresponding OBX data. The stars in the third plot represent the regime switching distribution based on simulations.

\begin{tabular}{l|c} 
Method & OBX \\
\hline Regime switching & 3762.4 \\
NIG & 3757.7 \\
Normal & 3688.2
\end{tabular}

TABLE 2. Loglikelihoods for the regime switching distribution (or rather the i.i.d. mixture distribution), the NIG distribution and the normal distribution for the logreturns of OBX displayed in Figure 3.

seems as though the regime switching distribution picks up slightly heavier tails than the NIG distribution. The normal distribution gives the least good fit to the data. 


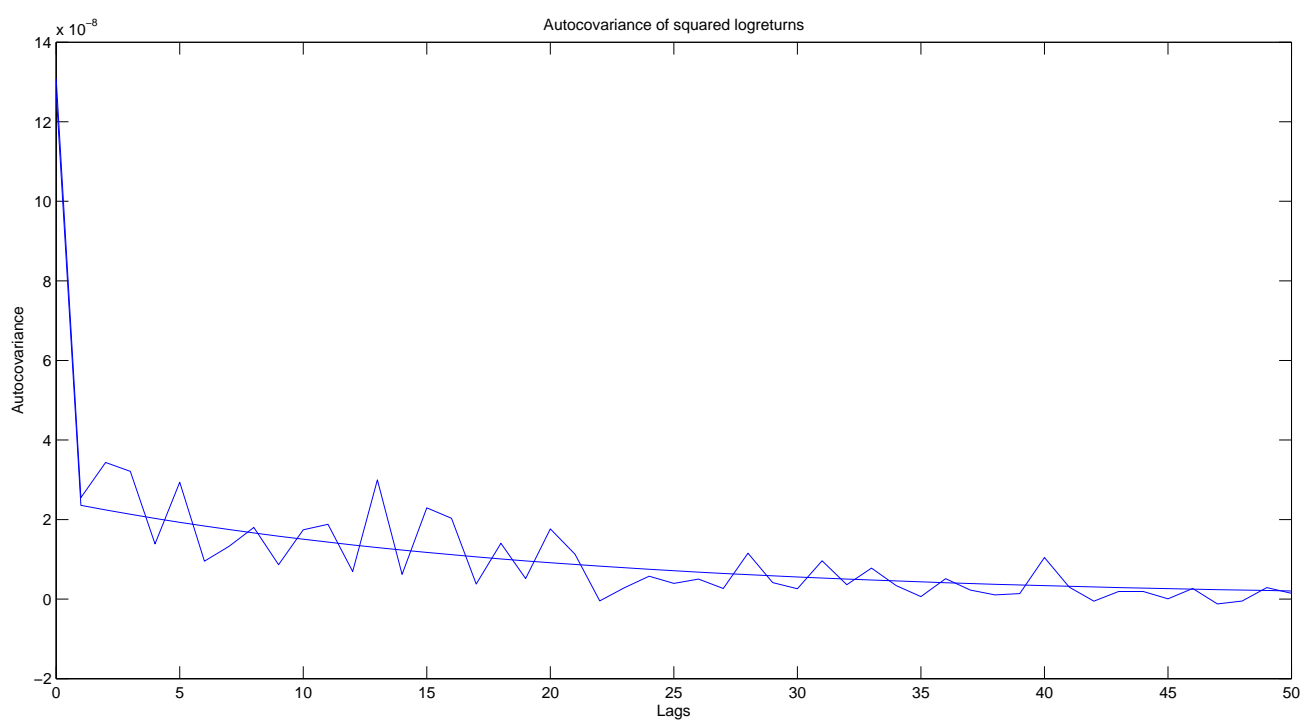

Figure 4. Autocovariance from the first 50 lags of squared logreturns from data and the regime switching model.

The transition probability matrix for the three regime switching model is given by:

$$
\boldsymbol{P}=\left[\begin{array}{lll}
0.3954 & 0.0102 & 0.5944 \\
0.0386 & 0.9612 & 0.0002 \\
0.4952 & 0.0094 & 0.4954
\end{array}\right]
$$

From this matrix, we see that the Markov chain might have a tendency to jump between state 1 and 3 , and only occasionally jump to the high volatility state number 2 . However, when the Markov chain is in state number 2 it tends to stay there longer than it does in the other states.

The matching of the autocovariance of squared logreturns has been done when estimating the $\boldsymbol{P}$ matrix as described in Section 3. In Figure 4 the autocovariance of squared logreturns from data and from the regime switching model based on Theorem 3.1 has been displayed. As we see, the autocovariance from the model seems to make a nice least squares line from the data. When the autocovariance of the squared logreturns has been matched, we should expect volatility clustering from the data and simulations from the regime switching model to be similar. In Figure 5 daily logreturns from the data and a random simulation has been plotted. From here we see that the structure in the volatility clustering is very similar for the two time series.

Having a nice fit to the logreturn distribution and having matched the autocovariance of squared logreturns, it is interesting to see a few simulations and compare these to the original data. In Figure 6 the OBX data has been plotted together with three random simulations from the regime switching model. As we see, the structure of the asset paths seem to be very similar. 

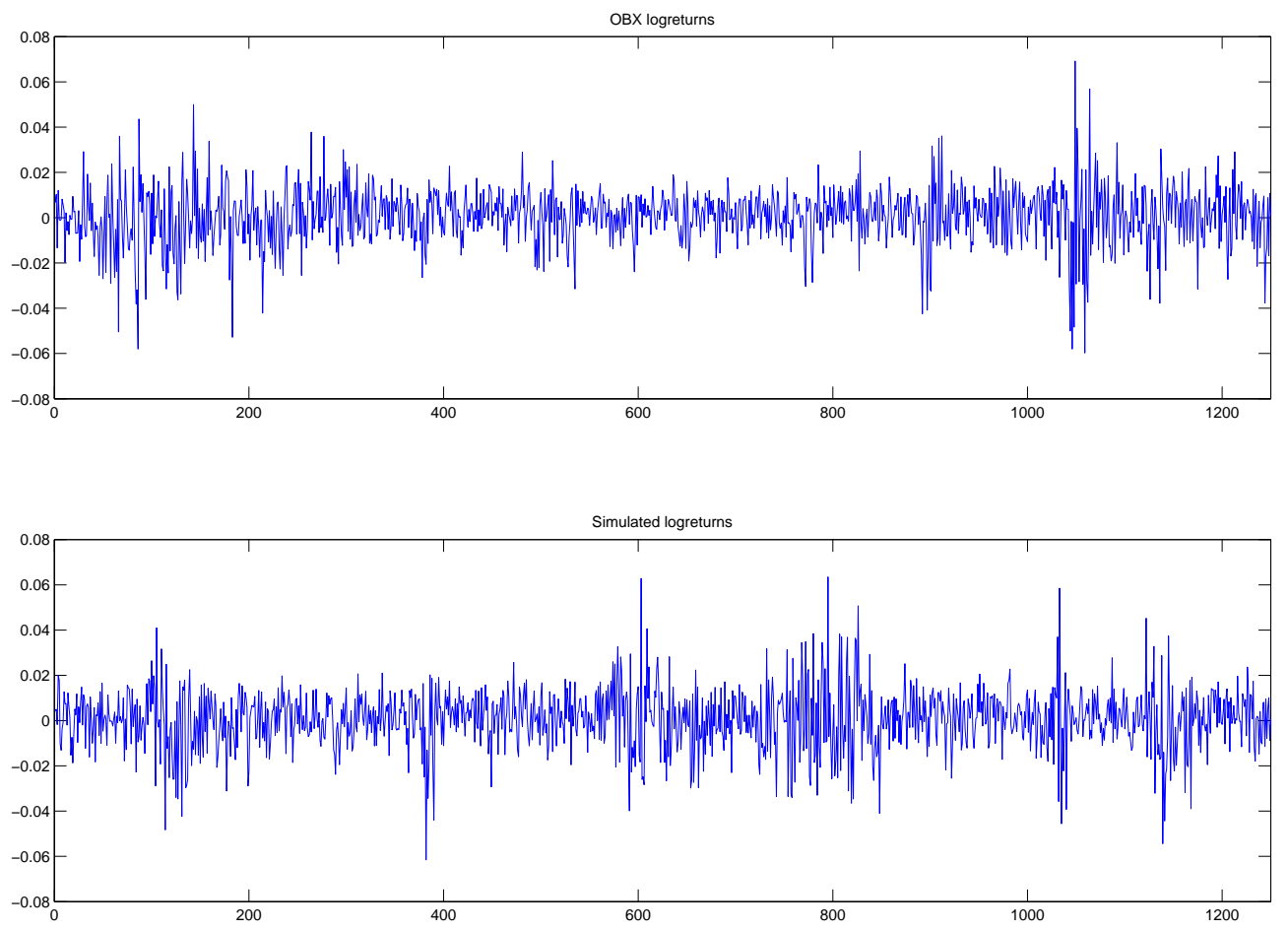

Figure 5. Daily logreturns from the OBX data and a random simulation, both time series displaying similar volatility clustering.

\begin{tabular}{|c|c|c|c|c|c|c|}
\hline & \multicolumn{3}{|c|}{ Regime switching } & \multicolumn{3}{c|}{ Black and Scholes } \\
\hline$K \backslash B$ & 161.26 & 227.23 & 293.20 & 161.26 & 227.23 & 293.20 \\
\hline 131.94 & 21.2456 & 2.9471 & 0.2308 & 22.3642 & 3.5036 & 0.1347 \\
175.92 & 3.8746 & 1.6313 & 0.1736 & $4.5235 *$ & 1.9243 & 0.0983 \\
219.90 & 0.4695 & 0.4669 & 0.1041 & $0.5018 *$ & 0.4956 & 0.0619 \\
\hline
\end{tabular}

TABLE 3. Option prices for different barriers $B$ and strikes $K$ based on the regime switching model in Section 4 compared to option prices from the closed form formula (4.2) in a Black and Scholes market. Prices marked by * have $B<K$, and is hence a pure European call price.

Prices for up-and-in call barrier options based on the methodology introduced in Section 4 is displayed in Table 3 for different barriers $B$ and strikes $K$. All the options have been priced with risk free daily interest rate $r=0.00016$, time to maturity $T=250$ days, initial asset value $S(0)=146,60$ and $10^{6}$ simulations from the regime switching model. It is interesting to see that the option prices for low barriers and strikes are cheaper under 


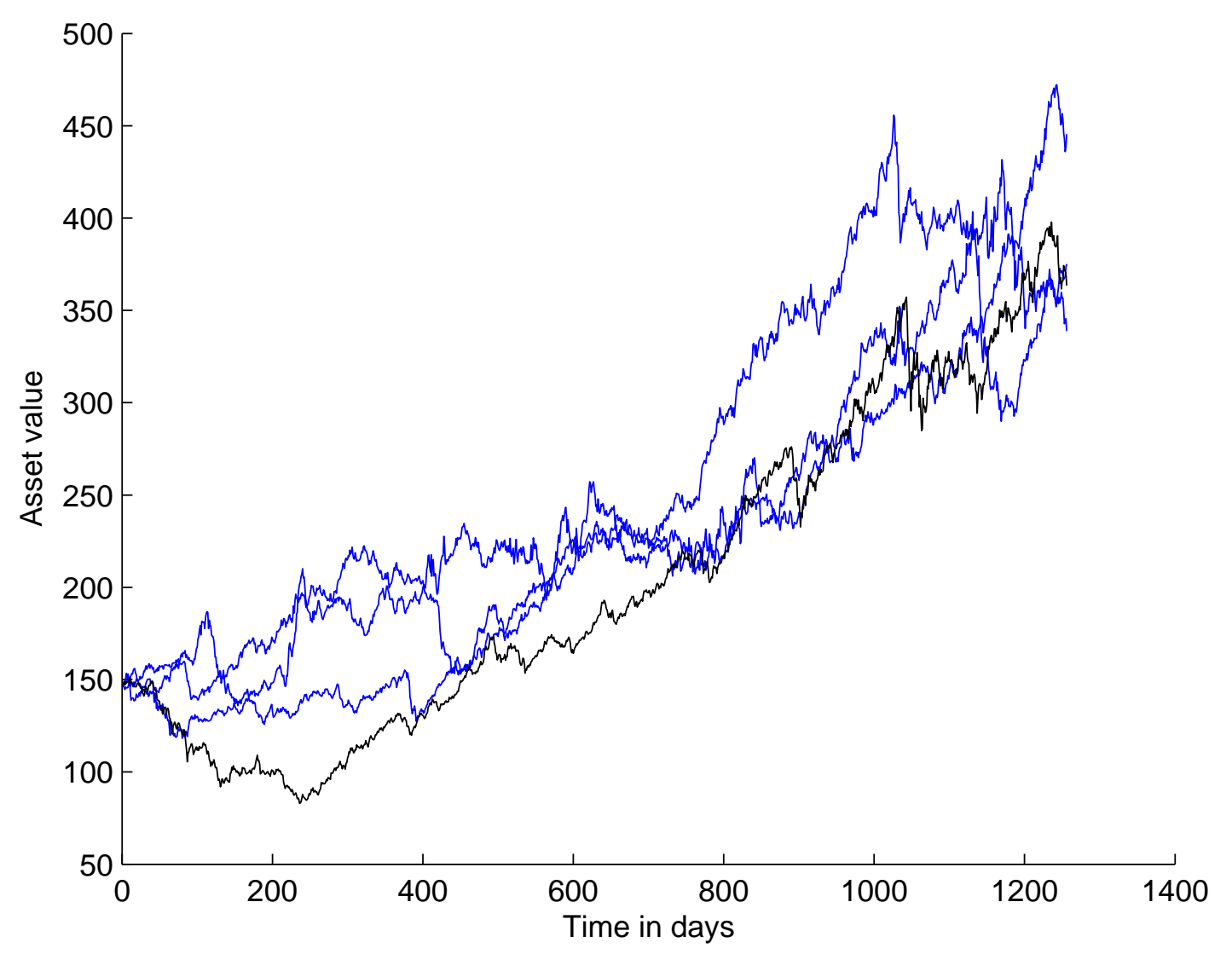

Figure 6. The OBX data versus time in black, and three simulations from the regime switching model in blue.

the regime switching model than under the Black and Scholes model. However, for higher barriers and strikes the option price is more expensive using the regime switching model. Looking at Figure 7, this phenomenon is quite intuitive. In this figure, we have plotted the logarithm of the probability density function of $S(T)$ under the risk neutral probability measure $\mathrm{Q}$ for the regime switching model and the Black and Scholes model. It is clear that the regime switching model has fatter tails, which induces higher prices for barriers and strikes in this tail. However, the Black and Scholes probability density function has higher mass for lower barriers and strikes, which gives a higher option price in this area.

\section{Conclusion}

A great advantage of the regime switching model is the intuitive model buildup. For e.g. three regimes, one may look at one regime as a normal market, one regime as a bull market and one regime as a bear market - all markets modeled by Brownian motion. This picks up skewness and kurtosis in financial logreturns in a nice way. We have seen that 


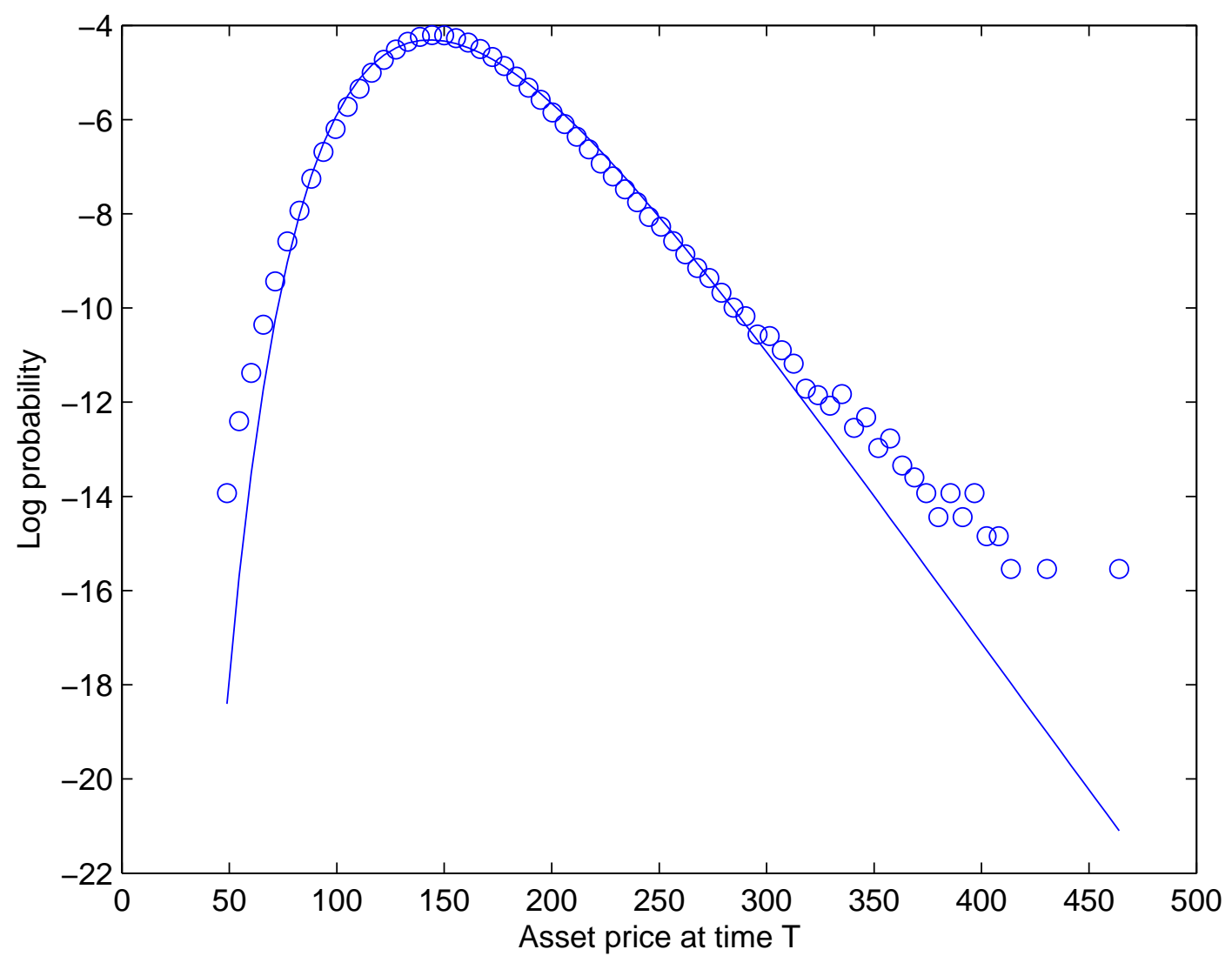

FIGURE 7. Logarithmic probability density function for $S(T)$ under the risk neutral measure $\mathrm{Q}$ from the regime switching model, marked by circles, and the Black and Scholes model, marked by the whole line.

the regime switching model gave at least as good a fit to Norwegian stock exchange index data as the popular NIG model, and much better fit than the traditional B\&S model.

In this paper we have introduced a new way to estimate parameters in a regime switching asset model to take volatility clustering into account. The volatility clustering may be particularly important in valuation of path dependent derivatives like barrier options where a cluster may lead the asset to cross a barrier. Further to this, we have seen that including probability of crossing the barrier between simulation points and improving efficiency and accuracy in simulations by importance sampling is easily done when applying the regime switching model.

For derivative pricing the regime switching model has an intuitive risk neutral probability measure. This is based on B\&S market price of risk, and it is therefore fruitful to compare option prices based on regime switching with option prices based on the B\&S model. Regarding the barrier option prices, we have seen that the regime switching model gives 
lower prices than the B\&S model for in the money strikes and barriers. However, the regime switching model picks up more risk in the tails of the maturity asset price distribution, which generates higher prices for fare out of the money options. This feature is tractable in real life pricing of derivatives, as this would straighten out the volatility smile used in $\mathrm{B} \& \mathrm{~S}$ pricing in practice.

\section{ACKNOWLEDGMENTS}

The author expresses great gratitude to Professor Fred Espen Benth for contributing with ideas and insightful knowledge in many discussions during the process of writing this paper. Also, thank is due to Professor Frank Proske for very valuable comments and suggestions of improvement.

\section{REFERENCES}

[1] Alexander, C. (2004). Normal mixture diffusion with uncertain volatility: Modelling short- and longterm smile effects, Journal of Banking \& Finance, 28, pp. 2957-2980.

[2] Barndorff-Nielsen, O. E. (1997). Normal inverse Gaussian distributions and stochastic volatility modeling, Scand. J. Statist., 24, pp. 1-13.

[3] Benth, F. E. (2004). Option Theory with Stochastic Analysis, Springer

[4] Bilmes, J. A. (1998). A Gentle Tutorial of the EM Algorithm and its Application to Parameter Estimation for Gaussian Mixture and Hidden Markov Models, ICSI TR-97-021.

[5] Black, F. and Scholes, M. (1973). The pricing of options and corporate liabilities, Journal of Political Economy, 3.

[6] Boldin, M. D. (1996). A Check on the Robustness of Hamilton's Markov Switching Model Approach to the Economic Analysis of the Business Cycle, Studies in Nonlinear Dynamics and Econometrics, 1(1), pp. 35-46.

[7] Bollen, N. P. B. (1998). Valuing Options in Regime Switching Models, Journal of Derivatives, 6, pp. 38-49.

[8] Brigo, D., Mercurio, F. and Rapisarda, F. (2004). Smile at the uncertainty, Risk, 17.

[9] Ching, W.-K., Siu T.-K. and Li, L.-M. (2007). Pricing Exotic Options under a High-Order Markovian Regime Switching Model, Journal of Appl. Math. and Decision Sciences, 2007, 15 pages.

[10] Chourdakis, K. (2002). Continuous Time Regime Switching Models and Applications in Estimating Processes with Stochastic Volatility and Jumps, University of London, Queen Mary, Working Paper no. 464 .

[11] Chourdakis, K. (2005). Lévy processes driven by stochastic volatility, Asia-Pacific Finan Markets, 12, pp. 333-352.

[12] Cont, R. and Tankov, P. (2004). Financial Modelling With Jump Processes, Chapman \& 6 Hall.

[13] Frühwirth-Schnatter, S. (2006). Finite Mixture and Markov Switching Models, Springer Series in Statistics.

[14] Hamilton, J. D. (1989). A New Approach to the Economic Analysis of Non-stationary Time Series, Econometrica, 57, pp. 357-384.

[15] Hardy, M. (2001). A Regime-Switching Model of Long-Term Stock Returns, North American Actuarial Journal, 5(2), pp. 41-53.

[16] Glasserman, P. (2004). Monte Carlo Methods in Financial Engineering, Springer.

[17] Hull, J. C. (2003). Options, Futures and Other Derivatives, Prentice Hall, Fifth Edition.

[18] Joshi, M. S. and Leung, T. (2007). Using Monte Carlo Simulation and Importance Sampling to Rapidly Obtain Jump-Diffusion Prices of Continuous Barrier Options, Journal Computational Finance, pp. 93105. 
[19] Øksendal, B. (2003). Stochastic Differential Equations, Springer, Sixth Edition.

[20] Rasmus, S (2006). Derivative Prices for Models Using Lévy Processes and Markov Switching, PhD Thesis Lund University.

[21] Timmermann, A. (2000). Moments of Markov switching models, Journal of Econometrics, 96, pp. 75111.

(Pål Nicolai Henriksen), Department of Mathematics, University of Oslo, P.O. Box 1053, Blindern, N-0316 Oslo, Norway

E-mail address: paalnhe@math.uio.no 